\title{
Fluctuation-Driven Magnetic Hard-Axis Ordering in Metallic Ferromagnets
}

\author{
F. Krüger, ${ }^{1,2}$ C. J. Pedder, ${ }^{1}$ and A. G. Green ${ }^{1}$ \\ ${ }^{1}$ London Centre for Nanotechnology, University College London, Gordon Street, London WC1H OAH, United Kingdom \\ ${ }^{2}$ ISIS Facility, Rutherford Appleton Laboratory, Chilton, Didcot, Oxfordshire OX11 OQX, United Kingdom
}

(Received 16 June 2014; revised manuscript received 18 August 2014; published 3 October 2014)

\begin{abstract}
We demonstrate that the interplay between soft electronic particle-hole fluctuations and magnetic anisotropies can drive ferromagnetic moments to point along a magnetic hard axis. As a proof of concept, we show this behavior explicitly for a generic two-band model with local Coulomb and Hund's interactions and a spin-orbit-induced easy plane anisotropy. The phase diagram is calculated within the fermionic quantum order-by-disorder approach, which is based on a self-consistent free-energy expansion around a magnetically ordered state with unspecified orientation. Quantum fluctuations render the transition of the easy-plane ferromagnet first order below a tricritical point. At even lower temperatures, directionally dependent transverse fluctuations dominate the magnetic anisotropy, and the moments flip to lie along the magnetic hard axis. We discuss our findings in the context of recent experiments that show this unusual ordering along the magnetic hard direction.
\end{abstract}

DOI: 10.1103/PhysRevLett.113.147001

Low-energy, electronic particle-hole fluctuations in metallic magnets can have drastic and often surprising effects. In itinerant ferromagnets (FMs), they generically render a priori continuous phase transitions first order at low temperatures [1-5]. In many systems, quantum phase transitions are preempted by the formation of superconducting [5-7], modulated magnetic [8], or unusual spin-glass phases $[9,10]$. Other metallic FMs show an unexpected ordering along the magnetic hard axis $[11,12]$.

It is well understood that the first-order behavior arises from the coupling of the magnetic order parameter to soft electronic particle-hole fluctuations, giving rise to nonanalytic terms in the free energy [13-16]. Because of this interplay between low-energy quantum fluctuations, metallic FMs are very susceptible towards the formation of incommensurate magnetic [17], spin nematic [14], or modulated superconducting states [18]. This spatial modulation is associated with deformations of the Fermi surface that enhance the phase space for low-energy particle-hole fluctuations. The phase reconstruction can therefore be viewed as a fermionic quantum order-by-disorder effect, which can be studied systematically by self-consistently calculating fluctuations around a whole class of possible broken-symmetry states [19-21].

The coupling to electronic quantum fluctuations can also have counterintuitive effects upon the direction of the magnetic order parameter. A notable example is the partially ordered phase of the helimagnet $\mathrm{MnSi}$, in which the spiral ordering vector rotates away from the latticefavored directions $[22,23]$. Similar effects are possible in homogeneous itinerant FMs. This is suggested by recent experiments that show unusual ordering of magnetic moments along hard magnetic directions [11,12].
PACS numbers: 74.40.Kb, 75.30.Gw, 75.50.Cc, 75.70.Tj

The first example is $\mathrm{YbRh}_{2} \mathrm{Si}_{2}$, which is a prototypical system for studying antiferromagnetic quantum criticality but exhibits strong FM fluctuations [24]. Interestingly, isoelectronic cobalt substitution for rhodium or hydrostatic pressure stabilizes FM order along the hard axis at low temperatures $[11,25]$. This behavior has been interpreted as a combined effect of magnetic frustration and classical fluctuations of the magnetic order parameter [26]. Electronic particle-hole fluctuations at low temperatures are potentially strong enough to drive a moment reorientation even in the absence of frustration. This is supported by recent experiments on the metallic $\mathrm{FM} \mathrm{YbNi}_{4} \mathrm{P}_{2}$ [12]. At very low temperatures-well below the Kondo temperature - this system displays a switch of magnetic response anisotropy similar to $\mathrm{YbRh}_{2} \mathrm{Si}_{2}$ and develops $\mathrm{FM}$ order along the hard direction.

In this Letter, we show that such a fluctuation-driven moment reorientation from the easy towards the hard direction is expected to occur in a large variety of itinerant FMs. The underlying mechanism has the same origin as the fluctuation-driven first-order behavior seen in nearly all itinerant FMs, irrespective of microscopic details [16].

The necessary physical ingredients are (i) two itinerant bands with different orbital character and sufficiently isotropic Fermi surfaces, (ii) a crystal-field splitting $\Delta$ that lifts the degeneracy between the orbitals, (iii) a spin-orbit (SO) coupling $\lambda$ between the bands that, in combination with (ii), generates magnetic anisotropy, and (iv) shortrange repulsive interactions that drive a FM instability. The interplay between the magnetic order-parameter and soft electronic particle-hole fluctuations gives rise to nonanalyticities in the free energy, e.g., an isotropic contribution $\delta F \sim m^{4} \ln \left[m^{2}+(T / \mu)^{2}\right]$ (in $d=3$ ) [13] that renders the FM transition first order at low temperatures. In the 
presence of anisotropy, such nonanalyticities couple to directional-dependent terms that will dominate the behavior at sufficiently low temperatures. To be more precise, at mean-field level, SO coupling leads to magnetic anisotropy $F_{\text {ani }} \sim\left(m_{\|}^{2}-m_{\perp}^{2}\right)$ with $m_{\|}$and $m_{\perp}$ the moment components along the hard and easy directions, respectively. Fluctuations produce a direction-dependent, nonanalytic free-energy contribution

$$
\delta F_{\mathrm{ani}} \simeq \frac{\lambda^{2}}{8 \Delta^{2}} m^{2}\left(m_{\|}^{2}-m_{\perp}^{2}\right) \ln (T / \mu),
$$

that competes with the mean-field anisotropy and dominates at sufficiently low temperatures. The switching of moments toward the magnetic hard axis is driven by the low-energy, transverse spin fluctuations-a "quantum Indian rope trick" [27].

Our starting point is a generic two-band model with local intraband Hubbard repulsion $U$, Hund's coupling $J_{H}$, and SO coupling $\lambda$ between the bands:

$$
\begin{aligned}
H= & \sum_{\alpha=1,2} \sum_{\nu=\uparrow, \downarrow} \int_{\mathbf{k}} \epsilon_{\alpha}(\mathbf{k}) c_{\mathbf{k} \alpha \nu}^{\dagger} c_{\mathbf{k} \alpha \nu}+U \sum_{\mathbf{r}, \alpha} \hat{n}_{\mathbf{r} \alpha \uparrow} \hat{n}_{\mathbf{r} \alpha \downarrow} \\
& -J_{H} \sum_{\mathbf{r}} \hat{\mathbf{s}}_{\mathbf{r} 1} \cdot \hat{\mathbf{s}}_{\mathbf{r} 2}+\frac{\lambda}{2} \int_{\mathbf{k}}\left(c_{\mathbf{k}, 1 \uparrow}^{\dagger} c_{\mathbf{k}, 2 \downarrow}-c_{\mathbf{k}, 1 \downarrow}^{\dagger} c_{\mathbf{k}, 2 \uparrow}+\text { H.c. }\right),
\end{aligned}
$$

where the operators $c_{\mathbf{k} \alpha \nu}^{\dagger}\left(c_{\mathbf{k} \alpha \nu}\right)$ create (annihilate) an electron with momentum $\mathbf{k}$ and spin $\nu$ in band $\alpha$ and $\hat{n}_{\mathbf{r} \alpha \nu}=c_{\mathbf{k} \alpha \nu}^{\dagger} c_{\mathbf{k} \alpha \nu}$ and $\hat{\mathbf{s}}_{\mathbf{r} \alpha}=\frac{1}{2} \sum_{\nu \nu^{\prime}} c_{\mathbf{r} \alpha \nu}^{\dagger} \sigma_{\nu \nu^{\prime}} c_{\mathbf{r} \alpha \nu^{\prime}}$ denote the occupation-number and electron-spin operators, respectively. Here $\sigma_{\nu \nu^{\prime}}=\left(\sigma_{x}, \sigma_{y}, \sigma_{z}\right)_{\nu \nu^{\prime}}$, with $\sigma_{i}$ the standard Pauli matrices. If the Fermi surfaces are sufficiently isotropic and centered around the $\Gamma$ point, we can approximate the electron dispersions by $\epsilon_{1}(\mathbf{k})=t k^{2}$ and $\epsilon_{2}(\mathbf{k})=\epsilon_{1}(\mathbf{k})+\Delta$, where $\Delta$ is the crystal-field splitting. For simplicity, we assume the band masses to be the same. Tight-binding corrections to the dispersion do not lead to magnetic anisotropies or qualitatively change the phase diagram as long as the system is far from instabilities due to nesting or van Hove singularities [19]. The SO term is of the standard form for an orbital multiplet that transforms as an $L=1$ angular momentum (e.g., the three $t_{2 g}$ orbitals) [30], projected onto the two lowest bands [31]. For simplicity, we neglect the local interband repulsion $V$, which only renormalizes $\mu$ and $\Delta$ but does not qualitatively change the physics of the model [32].

We first explain the mean-field behavior of the free energy. For sufficiently large $U$, the ground state of the system is a FM, and the directions of the magnetizations $\boldsymbol{m}_{1}$ and $\boldsymbol{m}_{2}$ of the two bands are locked together by the Hund coupling $J_{H}$. Without SO interaction $(\lambda=0)$, the meanfield free energy is independent of the direction of the total magnetization:

$$
\begin{aligned}
F_{\mathrm{mf}}^{(0)}= & \frac{1}{1-\left(\frac{J_{H}}{2 U}\right)^{2}}\left[U\left(\tilde{m}_{1}^{2}+\tilde{m}_{2}^{2}\right)-J_{H} \tilde{m}_{1} \tilde{m}_{2}\right] \\
& +\sum_{\alpha}\left(A_{\alpha} \tilde{m}_{\alpha}^{2}+B_{\alpha} \tilde{m}_{\alpha}^{4}+C_{\alpha} \tilde{m}_{\alpha}^{6}\right) .
\end{aligned}
$$

Here we have introduced the weighted moments $\tilde{m}_{\alpha}=m_{\alpha}+\left(J_{H} / 2 U\right) m_{\bar{\alpha}}$, with $\bar{\alpha}=2$ for $\alpha=1$ and vice versa. The coefficients in this Landau expansion are given by $A_{\alpha}=U^{2} \int_{\mathbf{k}} n_{F}^{\prime}\left[\epsilon_{\alpha}(\mathbf{k})\right], B_{\alpha}=\left(U^{4} / 12\right) \int_{\mathbf{k}} n_{F}^{\prime \prime \prime}\left[\epsilon_{\alpha}(\mathbf{k})\right]$, and $C_{\alpha}=\left(U^{6} / 360\right) \int_{\mathbf{k}} n_{F}^{(5)}\left[\epsilon_{\alpha}(\mathbf{k})\right]$ with $n_{F}$ the Fermi function. Because of the crystal-field splitting $\Delta$ between the two bands, we find two distinct magnetic transitions that are separated in temperature. Since we are interested in behavior near the paramagnetic state, for large enough $\Delta$ we approximate the free energy, keeping only the classical contributions to the $\alpha=2$ band. In this case, $\tilde{m}_{2}=\left(J_{H} / 2 U\right) \tilde{m}_{1}$.

The directional dependence arises from SO coupling, which we treat in the second-order perturbation theory. This is justified if the hybridization of the bands is small, $\lambda / 2 \Delta \ll 1$. We obtain

$$
F_{\mathrm{mf}}^{(\lambda)}=\frac{\lambda^{2}}{2 U^{2}} B_{1}\left(\mathcal{P}_{\|}-\mathcal{P}_{\perp}\right) \tilde{m}_{1} \tilde{m}_{2},
$$

where we have defined the projectors onto the $y$ axis $\mathcal{P}_{\|}=n_{y}^{2}$ and the $x z$ plane $\mathcal{P}_{\perp}=n_{x}^{2}+n_{z}^{2}=1-n_{y}^{2}$ in terms of a unit vector $\mathbf{n}$ which parametrizes the magnetization direction $\boldsymbol{m}_{\alpha}=m_{\alpha} \mathbf{n}$. Since $B_{1}>0$, we obtain an easyplane anisotropy at the mean-field level.

To compute the fluctuation contributions to the magnetic free energy, we employ the fermionic quantum order-bydisorder approach. The key idea is to self-consistently expand around a whole class of magnetically ordered, broken-symmetry states that are not favored at the mean-field level but potentially stabilized by fluctuations. In this particular case, we expand around all possible homogeneous states with magnetization direction $\mathbf{n}=(\sin \theta \cos \phi, \sin \theta \sin \phi, \cos \theta)$. Readers not interested in the technical details might jump to the final results, which are presented after Eq. (9).

We first express the action as a fermion coherent-state path integral over Grassmann fields $\Psi(\mathbf{r}, \tau)=$ $\left[\psi_{\uparrow}(\mathbf{r}, \tau), \psi_{\downarrow}(\mathbf{r}, \tau)\right]$. To decouple the interaction terms, we perform a Hubbard-Stratonovich transformation, introducing spin- and charge-fluctuation fields $\phi_{\alpha}(\mathbf{r}, \tau)$ and $\rho_{\alpha}(\mathbf{r}, \tau)$. This leads to the action

$$
\begin{aligned}
\mathcal{S}= & \int_{0}^{\beta} d \tau \int d^{3} \mathbf{r}\left\{U \sum_{\alpha}\left(\boldsymbol{\phi}_{\alpha}^{2}-\rho_{\alpha}^{2}\right)+J_{H} \boldsymbol{\phi}_{1} \cdot \boldsymbol{\phi}_{2}\right. \\
& +\sum_{\alpha} \bar{\Psi}_{\alpha}\left[\partial_{\tau}-t \nabla^{2}-\mu_{\alpha}+U\left(\rho_{\alpha}-\boldsymbol{\varphi}_{\alpha} \cdot \boldsymbol{\sigma}\right)\right] \Psi_{\alpha} \\
& \left.+i \frac{\lambda}{2}\left(\bar{\Psi}_{1} \sigma_{y} \Psi_{2}-\bar{\Psi}_{2} \sigma_{y} \Psi_{1}\right)\right\},
\end{aligned}
$$


where we have defined $\mu_{1}=\mu, \quad \mu_{2}=\mu-\Delta$, and $\boldsymbol{\varphi}_{\alpha}:=\phi_{\alpha}+\left(J_{H} / 2 U\right) \boldsymbol{\phi}_{\bar{\alpha}}$. The magnetizations of the bands are given by the zero-frequency components of the spin-fluctuation fields: $\boldsymbol{m}_{\alpha}=\boldsymbol{\phi}_{\alpha}(\mathbf{r}, \omega=0)$. The resulting free-fermion action in the presence of static magnetic order is easily diagonalized by a rotation to new fermion fields $\eta_{\alpha}=\exp \left(i \theta \sigma_{y} / 2\right) \exp \left(i \phi \sigma_{z} / 2\right) \Psi_{\alpha}$, leading to $\mathcal{S}_{0}=\sum_{\alpha \nu} \sum_{\omega} \int_{\mathbf{k}} G_{\alpha, \nu}^{-1} \bar{\eta}_{\alpha, \nu} \eta_{\alpha, \nu}$ with Green's function

$$
G_{\alpha \nu}(\mathbf{k}, \omega)=\left\{i \omega-\left[\epsilon_{\alpha}(\mathbf{k})-\nu U \tilde{m}_{\alpha}-\mu\right]\right\}^{-1} .
$$

In this new basis, the SO terms explicitly depend upon the moment direction $\mathbf{n}$. We perform the Gaussian integral over the fermion fields, keeping terms up to quadratic order in the finite-frequency fluctuation fields. We then integrate over the fluctuation fields to obtain

$$
\begin{aligned}
F_{\mathrm{fl}}= & -\frac{1}{2} U^{2} T \sum_{\alpha, \tilde{\omega}} \sum_{\nu_{1}, \nu_{2}} \int_{\mathbf{q}}\left\{\Pi_{\nu_{1}, \nu_{2}}^{(\alpha)} \Pi_{\bar{\nu}_{1}, \bar{\nu}_{2}}^{(\alpha)}\right. \\
& \left.+\lambda^{2} \Pi_{\nu_{1}, \nu_{2}}^{(\alpha)}\left(\mathcal{P}_{\|} \Gamma_{\bar{\nu}_{1}, \bar{\nu}_{2}, \bar{\nu}_{2}}^{(\alpha)}+\mathcal{P}_{\perp} \Gamma_{\bar{\nu}_{1}, \bar{\nu}_{2}, \nu_{2}}^{(\alpha)}\right)\right\}+\cdots,
\end{aligned}
$$

where, as before, we keep only terms up to quadratic order in $\lambda$. In terms of the fermionic Green functions (6), the fermionic bubble diagrams are given by

$$
\begin{gathered}
\Pi_{\nu_{1} \nu_{2}}^{(\alpha)}(q)=T \sum_{\omega} \int_{\mathbf{k}} G_{\alpha \nu_{1}}(k+q) G_{\alpha \nu_{2}}(k), \\
\Gamma_{\nu_{1} \nu_{2} \nu_{3}}^{(\alpha)}(q)=T \sum_{\omega} \int_{\mathbf{k}} G_{\alpha \nu_{1}}(k+q) G_{\alpha \nu_{2}}^{2}(k) G_{\bar{\alpha}, \nu_{3}}(k),
\end{gathered}
$$

where we have defined $k=(\mathbf{k}, \omega)$ for brevity.

For $\lambda=0$, the bands decouple and $F_{\mathrm{fl}}(7)$ reduces to the expression for the single-band case [17,19]. After summation over Matsubara frequencies and resummation of the leading divergencies in temperature to all orders in the magnetization, we obtain [21]

$$
F_{\mathrm{fl}}^{(0)}=\frac{c_{1}}{2} \frac{U^{6}}{\mu^{5}} \tilde{m}_{1}^{4} \ln \left(\frac{\kappa U^{2} \tilde{m}_{1}^{2}+T^{2}}{\mu^{2}}\right)-c_{2} \frac{U^{4}}{\mu^{3}} \tilde{m}_{1}^{2},
$$

in agreement with the result of Belitz, Kirkpatrick, and Vojta [13]. We have neglected the fluctuation corrections for the second band, which is far from a magnetic instability, and defined $c_{1}=(16 / 3) \sqrt{2}(2 \pi)^{-6}(\mu / t)^{9 / 2}$ and $c_{2}=(1+\ln 2) c_{1}$. Since fluctuations give rise to a $\ln T$ contribution to the $m^{4}$ coefficient, the transitions turn first order below a certain tricritical temperature $T_{c}$.

Although the fluctuation terms of the order of $\lambda^{2}$ appear to be much harder to calculate, we can use a trick to rewrite $\Gamma_{\nu_{1} \nu_{2} \nu_{3}}^{(\alpha)}(q)$ in terms of a generalized derivative of $\Pi_{\nu_{1} \nu_{2}}^{(\alpha)}(q)$ [33]. We note that the dominant directional-dependent fluctuation terms originate from transversal spin fluctuations and are proportional to a derivative of $F_{\mathrm{fl}}^{(0)}$ :

$$
F_{\mathrm{fl}}^{(\lambda)}=\frac{\lambda^{2}}{8 \Delta^{2}}\left(\mathcal{P}_{\|}-\mathcal{P}_{\perp}\right) \tilde{m}_{2} \partial_{\tilde{m}_{1}} F_{\mathrm{fl}}^{(0)}
$$

Since the magnetization of the system (global minimum of $\left.F_{\mathrm{mf}}+F_{\mathrm{fl}}\right)$ is always smaller than the value at which the function $F_{\mathrm{fl}}^{(0)}$ has its minimum, the derivative in Eq. (11) is negative. Hence, the directionally dependent fluctuation terms compete with the mean-field anisotropy and could potentially stabilize FM order along the hard axis at sufficiently low temperatures.

We calculate the phase diagram by minimizing the free energy $F=F_{\mathrm{mf}}^{(0)}+F_{\mathrm{mf}}^{(\lambda)}+F_{\mathrm{fl}}^{(0)}+F_{\mathrm{fl}}^{(\lambda)}$. For small SO coupling $\lambda$, we can approximate $\tilde{m}_{2} \approx\left(J_{H} / 2 U\right) \tilde{m}_{1}$. The free energy then becomes a function of $\tilde{m}_{1}$, which to leading order in $J_{H}$ is the total magnetization of the system. In Fig. 1, the phase diagram is shown as a function of temperature and inverse interaction strength. Because of the nonanalytic fluctuation correction $F_{\mathrm{fl}}^{(0)}$, the transition of the easy-plane FM turns first order at temperatures below the tricritical point $P$. At the point $Q$, located at a lower temperature, we find a crossing between the first-order lines calculated from the free energies $F_{\perp}$ and $F_{\|}$for moments in the easy plane and along the hard direction, respectively. Consequently, there is a region below the point $Q$ where fluctuations stabilize magnetic order along

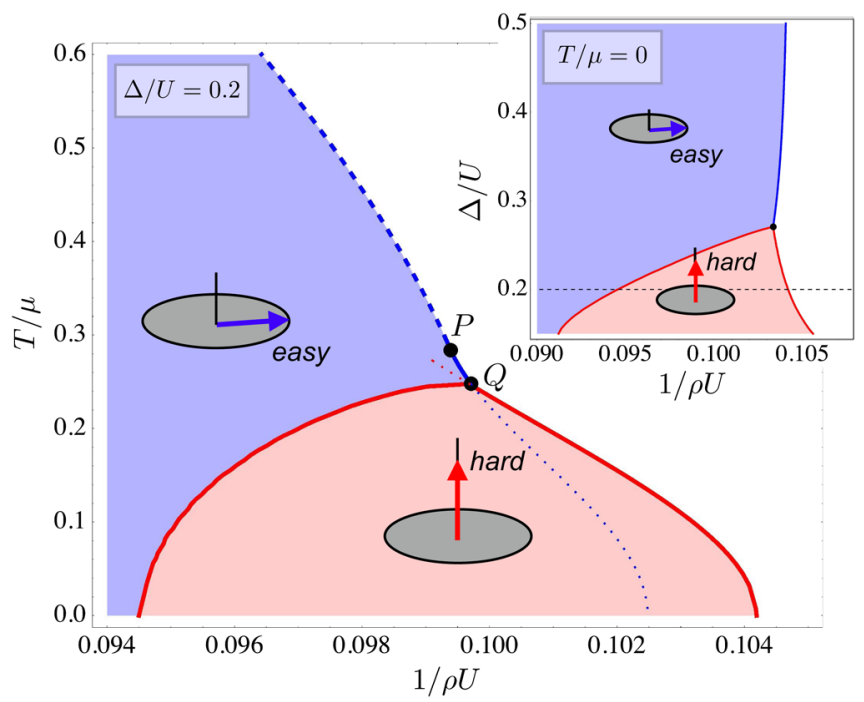

FIG. 1 (color online). Phase diagram as a function of temperature $T / \mu$ and inverse interaction strength $1 / \rho U$ ( $\rho$ is proportional to the density of states of band $\alpha=1$ at the Fermi level) for $J_{H} / 2 U=0.3, \lambda / U=0.2$, and $\Delta / U=0.2$. At the tricritical point $P$, the nature of the phase transition of the easy-axis FM (blue region) changes from second to first order, indicated by dashed and solid lines, respectively. In the red region below the point $Q$, fluctuations stabilize FM order along the hard axis. This region is enclosed by first-order transitions. The inset shows the $T=0$ phase diagram for the same parameters as a function of inverse interaction strength $1 / \rho U$ and crystal-field splitting $\Delta / U$. 
the hard axis. As one should expect, the transition at which the moments flip is first order.

Note that the region of FM order along the hard axis shrinks with increasing band splitting $\Delta$ and is completely suppressed beyond a critical value $\Delta_{c}$ (see the inset in Fig. 1). Since the competing directional-dependent terms $F_{\mathrm{mf}}^{(\lambda)}$ and $F_{\mathrm{fl}}^{(\lambda)}$ are of the same order in the Hund interaction $J_{H}$ and SO coupling $\lambda$, these parameters have only a small effect on the stability of the hard-axis FM. The limit $\Delta \rightarrow 0$ where the two bands become equivalent is not accessible by a perturbative treatment of the $\mathrm{SO}$ coupling, requiring $\lambda / 2 \Delta \ll 1$. As $\Delta \rightarrow 0$, the SO-induced hybridization of the bands increases, and the interband fluctuations become increasingly important. This would therefore require the insertion of an increasing number of SO terms in the fermion bubble diagrams. Each insertion increases the order by $\lambda / 2 \Delta$.

Depending upon the value of $U$, which can be viewed as a proxy for tuning parameters like pressure or chemical doping, there are three possible scenarios of phase transitions as a function of decreasing temperature.

(i) For sufficiently large $U$, we expect a single transition from a paramagnet to an easy-plane FM that is stable down to $T=0$. Depending on the separation of $P$ and $Q$, this transition could be continuous or first order.

(ii) Over an intermediate range of $U$ we expect a sequence of two transitions, first from a paramagnet into an easy-plane FM (first or second order) and then at lower temperatures into a FM state with moments along the hard direction. The transition at which the moment direction switches is always discontinuous.

(iii) Decreasing $U$ further, we find a single first-order transition from a paramagnet into a hard-axis FM.

To make contact with experiments, we calculate the magnetic susceptibilities $\chi_{\perp}$ and $\chi_{\|}$for fields along the easy and hard directions. In Fig. 2, the evolution of the susceptibilities with temperature is shown for different values of the electron repulsion $U$. Figures 2(a) and 2(b) correspond to case (i) with single continuous transitions into the easy-plane FM. At the transition, the easy-plane susceptibility $\chi_{\perp}$ diverges. The proximity of the system to a fluctuation-driven reorientation of the moments is apparent from the increase in the hard-axis susceptibility as the temperature is reduced. Reducing $U$, this increase becomes more pronounced. Case (ii) is shown in Fig. 2(c). At the first-order transition between the two FM states, we find an inversion of $\chi_{\perp}$ and $\chi_{\|}$, characteristic of the switching of the moment direction.

Finally, Fig. 2(d) shows the temperature dependence of the susceptibilities in regime (iii) where the system exhibits a direct transition from a paramagnet to a FM state with moments along the hard direction, exactly as it has been observed experimentally [11,12]. We find a similar characteristic crossing of susceptibilities at a temperature $T^{*}$ slightly above the ordering temperature $T_{c}$. As we increase

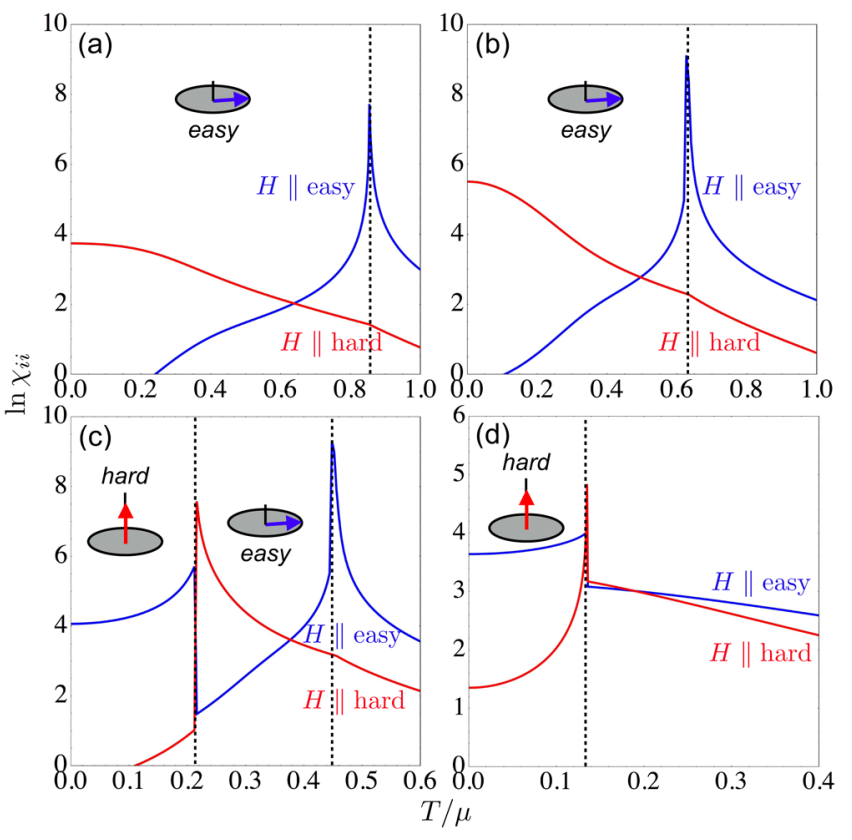

FIG. 2 (color online). Logarithm of the magnetic susceptibilities $\chi_{i i}=\partial m_{i} / \partial h_{i}$ for fields in the easy plane (blue line) and along the hard axis (red line) as a function of temperature for decreasing values of the electron repulsion: $1 /(\rho U)=0.093$ (a), 0.0945 (b), 0.098 (c), and 0.103 (d).

$U$ and move closer towards the point $Q$ (see Fig. 1), the temperatures $T^{*}$ and $T_{c}$ merge.

Discussion and conclusions.-We have described a generic mechanism whereby soft electronic particle-hole fluctuations drive unexpected magnetic behavior in itinerant FMs with magnetic anisotropy. At low temperatures, these systems can magnetize along directions that are unfavorable at higher temperatures. As a proof of principle, we have explicitly demonstrated this for a two-band model with an easy-plane anisotropy generated by SO coupling. At sufficiently low temperatures, the moments switch to the magnetic hard axis in order to maximize the phase space for transverse spin fluctuations.

Related phase transitions are also possible. Quantum fluctuations could also stabilize a modulated spiral state which preempts the first-order transition into the homogeneous FM [17,19]. Since planar spirals are compatible with magnetic easy-plane anisotropies, one might find systems that show spiral order which then becomes unstable toward a hard-axis FM at even lower temperatures. For a tetragonal system with magnetic easy-axis anisotropy, we expect a similar moment reorientation as for the easyplane case studied in this Letter. Simply because of flipping the moments into the hard plane, the easy direction becomes available for transverse spin fluctuations. Orthorhombic systems with three inequivalent magnetic directions might offer even more interesting phase behavior with a two-step moment reorientation. 
With recent experimental advances, it is now possible to study the phase reconstruction down to extremely low temperatures. There are several materials in which FM ordering along a hard magnetic direction has been discovered. The most promising is $\mathrm{YbNi}_{4} \mathrm{P}_{2}$ [12] because of its close proximity to a quantum phase transition. The observed crossing of susceptibilities slightly above the ordering temperature is in qualitative agreement with our prediction based on a simple itinerant model. However, the measured critical exponents are inconsistent with a pure itinerant model [12]. In fact, $\mathrm{YbNi}_{4} \mathrm{P}_{2}$ exhibits quasi-onedimensional spin chains, which couple to the conduction electrons, and is a Kondo-lattice system with strong interactions between conduction and localized $f$ electrons. While the nonanalytic free-energy corrections are expected to have similar effects in systems with local moments [16], a quantitative analysis based on a realistic microscopic model for $\mathrm{YbNi}_{4} \mathrm{P}_{2}$ would be desirable.

We point out that the suggested mechanism for moment reorientation does not require frustration. It is expected to be generic, since it stems from the same nonanalytic freeenergy corrections that are responsible for fluctuationinduced first-order behavior in practically all itinerant FMs, irrespective of microscopic details. It is desirable to go beyond our simple model to study the effects of realistic band structures of specific materials, longer-range interactions, frustration, the coupling to local moments, and potentially even Kondo physics. These extra ingredients coupled with electronic low-energy fluctuations might yield a plethora of novel and exciting ordering phenomena.

The authors benefited from stimulating discussions with M. Brando, A. Steppke, and M. Vojta. This work has been supported by the EPSRC through Grant No. EP/I004831/2.

[1] C. Pfleiderer, S. R. Julian, and G. G. Lonzarich, Nature (London) 414, 427 (2001).

[2] Y. J. Uemura et al., Nat. Phys. 3, 29 (2007).

[3] M. Otero-Leal, F. Rivadulla, M. Garcia-Hernandez, A. Pineiro, V. Pardo, D. Baldomir, and J. Rivas, Phys. Rev. B 78, 180415(R) (2008).

[4] V. Taufour, D. Aoki, G. Knebel, and J. Flouquet, Phys. Rev. Lett. 105, 217201 (2010).

[5] E. A. Yelland, J. M. Barraclough, W. Wang, K. V. Kamenev, and A. D. Huxley, Nat. Phys. 7, 890 (2011).

[6] S. S. Saxena et al., Nature (London) 406, 587 (2000).

[7] E. Hassinger, D. Aoki, G. Knebel, and J. Flouquet, J. Phys. Soc. Jpn. 77, 073703 (2008).

[8] M. Brando, W. J. Duncan, D. Moroni-Klementowicz, C. Albrecht, D. Grüner, R. Ballou, and F. M. Grosche, Phys. Rev. Lett. 101, 026401 (2008).

[9] T. Westerkamp, M. Deppe, R. Küchler, M. Brando, C. Geibel, P. Gegenwart, A. P. Pikul, and F. Steglich, Phys. Rev. Lett. 102, 206404 (2009).

[10] S. Lausberg et al., Phys. Rev. Lett. 109, 216402 (2012).

[11] S. Lausberg et al., Phys. Rev. Lett. 110, 256402 (2013).
[12] A. Steppke et al., Science 339, 933 (2013).

[13] D. Belitz, T. R. Kirkpatrick, and T. Vojta, Phys. Rev. Lett. 82, 4707 (1999).

[14] A. V. Chubukov, C. Pépin, and J. Rech, Phys. Rev. Lett. 92, 147003 (2004).

[15] D. Belitz, T. R. Kirkpatrick, and T. Vojta, Rev. Mod. Phys. 77, 579 (2005).

[16] T. R. Kirkpatrick and D. Belitz, Phys. Rev. B 85, 134451 (2012).

[17] G. J. Conduit, A. G. Green, and B. D. Simons, Phys. Rev. Lett. 103, 207201 (2009).

[18] G. J. Conduit, C. J. Pedder, and A. G. Green, Phys. Rev. B 87, 121112 (2013).

[19] U. Karahasanovic, F. Krüger, and A. G. Green, Phys. Rev. B 85, 165111 (2012).

[20] S. J. Thomson, F. Krüger, and A. G. Green, Phys. Rev. B 87, 224203 (2013).

[21] C. Pedder, F. Krüger, and A. G. Green, Phys. Rev. B 88, 165109 (2013).

[22] C. Pfleiderer, D. Reznik, L. Pintschovius, H. v. Löhneysen, M. Garst, and A. Rosch, Nature (London) 427, 227 (2004).

[23] F. Krüger, U. Karahasanovic, and A. G. Green, Phys. Rev. Lett. 108, 067003 (2012).

[24] K. Ishida, K. Okamoto, Y. Kawasaki, Y. Kitaoka, O. Trovarelli, C. Geibel, and F. Steglich, Phys. Rev. Lett. 89, 107202 (2002).

[25] C. Klingner et al., Phys. Rev. B 83, 144405 (2011).

[26] E. C. Andrade, M. Brando, C. Geibel, and M. Vojta, Phys. Rev. B 90, 075138 (2014).

[27] In the extreme version of the Indian rope trick, a rope rises from a basket and a helper of the Fakir then climbs up the rope. Finally, the helper would descend or even disappear from view. This is either a hoax or true magic. It is, however, possible to perform an Indian rope trick with a chain of inverted pendula, stabilized by vibrations of the base point $[28,29]$.

[28] D. J. Acheson, Proc. R. Soc. A 443, 239 (1993).

[29] D. J. Acheson and T. Mullin, Nature (London) 366, 215 (1993).

[30] E. J. Rozbicki, J. F. Annett, J.-R. Souquet, and A. P. Mackenzie, J. Phys. Condens. Matter 23, 094201 (2011).

[31] I. Eremin, D. Manske, and K. H. Bennemann, Phys. Rev. B 65, 220502(R) (2002).

[32] W. Lv and P. Phillips, Phys. Rev. B 84, 174512 (2011).

[33] To compute the $\Gamma_{\nu_{1}, \nu_{2}, \nu_{3}}^{(1)}$ term, we write it as

$$
\begin{aligned}
\Gamma_{\nu_{1} \nu_{2} \nu_{3}}^{(1)} & =\frac{D_{\nu_{1} \nu_{2}}^{(1)} \Pi_{\nu_{1} \nu_{2}}^{(1)}-\frac{\left(\Pi_{\nu_{1} \nu_{2}}^{(1)}-\tilde{\Pi}_{\nu_{1} \nu_{2}}\right)}{2 \zeta_{\nu_{2} \nu_{3}}},}{\zeta_{\nu_{2} \nu_{3}}^{2}}, \\
\zeta_{\nu_{2} \nu_{3}} & =\Delta+U\left(\nu_{2} \tilde{m}_{1}-\nu_{3} \tilde{m}_{2}\right),
\end{aligned}
$$

where the derivative operators $D_{\nu \nu}^{(1)}=\partial_{\mu_{1}}$ and $D_{\nu \bar{\nu}}^{(1)}=$ $\partial_{\mu_{1}}-(\nu / U) \partial_{\tilde{m}_{1}}$ and $\tilde{\Pi}_{\nu_{1} \nu_{2}}(q)=T \sum_{\omega} \int_{\mathbf{k}} G_{1, \nu_{1}}(k+q) G_{2, \nu_{2}}(k)$. Substituting into the fluctuation term gives

$$
F_{\mathrm{fl}}^{(\lambda)} \approx-\frac{\lambda^{2} U^{2} T}{4 \Delta^{2}}\left(\mathcal{P}_{\|}-\mathcal{P}_{\perp}\right) \tilde{m}_{2} \partial_{\tilde{m}_{1}} \sum_{\tilde{\omega}} \int_{\mathbf{q}} \Pi_{+-}^{(1)} \Pi_{-+}^{(1)},
$$

which shows that the dominant terms originate from transverse spin fluctuations and leads to the result given in the text. 